\title{
Measurement of Very Low Density and Low Density Lipoprotein Apolipoprotein (Apo) B-100 and High Density Lipoprotein Apo A-I Production in Human Subjects Using Deuterated Leucine Effect of Fasting and Feeding
}

Jeffrey S. Cohn, David A. Wagner, Susan D. Cohn, John S. Millar, and Ernst J. Schaefer

Lipid Metabolism Laboratory, United States Department of Agriculture Human Nutrition Research Center on Aging

at Tufts University, Boston, Massachusetts 02111

\begin{abstract}
Six normolipidemic male subjects, after an 8-h overnight fast, were given a bolus injection and then a 15-h constant intravenous infusion of $\left[D_{3}\right] \mathrm{L}$-leucine. Subjects were studied in the fasted state and on a second occasion in the fed state (small, physiological meals were given every hour for 15 h). Apolipoproteins were isolated by preparative gradient gel electrophoresis from plasma lipoproteins separated by sequential ultracentrifugation. Incorporation of $\left[\mathrm{D}_{\mathbf{3}}\right] \mathrm{L}$-leucine into apolipoproteins was monitored by negative ionization, gas chromatography-mass spectrometry. Production rates were determined by multiplying plasma apolipoprotein pool sizes by fractional production rates (calculated as the rate of isotopic enrichment [IE] of each protein as a fraction of IE achieved by VLDL $(d<1.006 \mathrm{~g} / \mathrm{ml})$ apo B-100 at plateau. VLDL apo B-100 production was greater, and LDL $(1.019<d<1.063$ $\mathrm{g} / \mathrm{ml}$ ) apo B-100 production was less in the fed compared with the fasted state $(9.9 \pm 1.7$ vs. $6.4 \pm 1.7 \mathrm{mg} / \mathrm{kg}$ per $\mathrm{d}, P<0.01$, and $8.9 \pm 1.2$ vs. $13.1 \pm 1.2 \mathrm{mg} / \mathrm{kg}$ per $\mathrm{d}, P<0.05$, respectively). No mean change was observed in high density lipoprotein apo A-I production. We conclude that: $(a)$ this stable isotope, endogenous-labeling technique, for the first time allows for the in vivo measurement of apolipoprotein production in the fasted and fed state; and (b) since LDL apo B-100 production was greater than VLDL apo B-100 production in the fasted state, this study provides in vivo evidence that $L D L$ apo $B-100$ can be produced independently of VLDL apo B-100 in normolipidemic subjects. (J. Clin. Invest. 1990. 85:804-811.) stable isotope $\bullet$ postprandial triglyceridemia $\bullet$ apolipoprotein kinetics
\end{abstract}

\section{Introduction}

Plasma lipoproteins play a central role in the development of atherosclerosis. Elevated levels of LDL and decreased levels of HDL have repeatedly been associated with an increased risk of

Dr. Cohn's present address is Biochemistry Department, Royal Prince Alfred Hospital, Camperdown 2050, Sydney, Australia. Dr. Wagner's address is Shriner's Burn Institute, 51 Blossom St., Boston, MA 02114.

Address correspondence to Dr. Ernst J. Schaefer, Lipid Metabolism Laboratory, USDA Human Nutrition Research Center on Aging at Tufts University, 711 Washington St., Boston, MA 02111.

Received for publication 11 October 1988 and in revised form 6 October 1989.

J. Clin. Invest.

(c) The American Society for Clinical Investigation, Inc.

0021-9738/90/03/0804/08 \$2.00

Volume 85, March 1990, 804-811 coronary heart disease (1). Although numerous mechanisms control the circulating levels of these lipoproteins, one important determinant is the rate of production (synthesis and secretion) of their component apolipoproteins.

The measurement of apolipoprotein production in humans has predominantly involved the use of radioactive iodine-labeled lipoproteins (2). Lipoproteins and/or purified apolipoproteins have been isolated, radiolabeled, and reinjected as tracers, and their residence time in plasma has been determined by stochastic or multicompartmental analysis of plasma radioactivity decay curves. This approach, however, is not ideal for several reasons: (a) lipoproteins and apolipoproteins can be modified during isolation and radioiodination, which potentially affects their metabolic behavior in vivo; $(b)$ a steady-state condition must be assumed, where production and clearance rates are taken to be equal, an assumption that may not always be physiologically accurate; and (c) studies cannot be undertaken in young children or pregnant women, nor can multiple studies be undertaken in the same volunteer, due to exposure to potentially hazardous levels of radioactivity.

To circumvent some of these problems, Eaton et al. (3) and Fisher et al. (4) have measured apo B production by labeling apolipoproteins in vivo with intravenously injected $\left[{ }^{75} \mathrm{Se}\right]$ selenomethionine or $\left[{ }^{3} \mathrm{H}\right]$ leucine, respectively. More recently, Cryer et al. used a priming dose followed by a constant intravenous infusion of the stable isotope $\left[{ }^{15} \mathrm{~N}\right]$ glycine to measure VLDL apo B production (5). The rate of incorporation of $\left[{ }^{15} \mathrm{~N}\right]$ glycine into VLDL apo $\mathrm{B}$ was measured relative to the plateau in $\left[{ }^{15} \mathrm{~N}\right]$ glycine enrichment achieved by urinary hippurate, which was used as a measure of the hepatic glycine precursor pool. We have further developed and simplified the stable isotope measurement of apolipoprotein production by using a constant infusion of $\left[\mathrm{D}_{3}\right] \mathrm{L}$-leucine (deuterated leucine). By taking the plateau enrichment achieved by VLDL apo B-100 (the high molecular weight form of apo B, which is predominantly synthesized in the liver) as a measure of the hepatic leucine precursor pool, we have avoided the need to analyze urine samples. In this paper we describe our methodology and report the rates of production of VLDL apo B-100, LDL apo B-100, and HDL apo A-I, as measured in six male subjects in the fasted and fed state.

\section{Methods}

Subjects. Six male subjects who had no evidence or family history of hepatic, renal, thyroid, or cardiac dysfunction were recruited from the Boston area. They did not smoke or take medications known to affect plasma lipid levels. Subjects ranged in age from 24 to $37 \mathrm{yr}$ (mean age, $28 \pm 5 \mathrm{yr})$. They were of normal height $(175 \pm 3 \mathrm{~cm})$ and weight $(72.7 \pm 2.8 \mathrm{~kg}$ ), and they had normal fasting plasma lipid levels (choles- 
terol, $160 \pm 8 \mathrm{mg} / \mathrm{dl}$; triglyceride, $65 \pm 7 \mathrm{mg} / \mathrm{dl}$; HDL cholesterol, $46 \pm 4$ $\mathrm{mg} / \mathrm{dl}$ ). Four subjects had an apo $E 3 / 3$ phenotype and two had an apo E 4/3 phenotype (6). The experimental protocol was approved by the Human Investigation Review Committee of the New England Medical Center and Tufts University.

Experimental protocol. Subjects resided in the Metabolic Research Unit of the USDA Human Nutrition Research Center for the 10-d duration of the study. During this period they consumed a normal weight-maintaining diet and underwent two constant infusion experiments, one in the fasted state and one in the fed state. The first infusion experiment was carried out on day 6 and the second on day 9. The order of the experiments was randomized; four subjects did the experiment in the fasted state first and the experiment in the fed state second, while the remaining two subjects did the fed experiment first and the fasted experiment second.

The protocols for the constant infusion experiments are shown diagrammatically in Fig. 1. Each experiment was begun at 6 a.m. after an 8-h overnight fast. For the fed experiment, a large breakfast was given at 6 a.m., which contained $4 / 23$ of the prescribed amount given daily ( $40 \mathrm{cal} / \mathrm{kg}$ per d). At each hour thereafter, smaller meals were given, which contained $1 / 23$ of the prescribed daily amount. The smaller meals were identical and contained whole foods including spaghetti, meat, bread, butter, and milk. Each portion was carefully weighed and contained $45 \%$ carbohydrate, $15 \%$ protein, $40 \%$ fat ( $17 \%$ saturated, $17 \%$ monounsaturated, $6 \%$ polyunsaturated) by calories and 180 $\mathrm{mg} / 1,000$ cal cholesterol. 19 smaller, hourly meals were fed during the course of the experiment. At 11 a.m. ( $5 \mathrm{~h}$ after the first meal) each subject was given a bolus injection $(10 \mu \mathrm{mol} / \mathrm{kg})$ of $\left[\mathrm{D}_{3}\right] \mathrm{L}$-leucine (dissolved in $0.015 \mathrm{M} \mathrm{NaCl}$ ) via an intravenous line attached to a forearm vein. This was immediately followed by a constant infusion $(50 \mathrm{ml} / \mathrm{h})$ of $\left[\mathrm{D}_{3}\right] \mathrm{L}$-leucine $(10 \mu \mathrm{mol} / \mathrm{kg}$ per $\mathrm{h})$ dissolved in $0.015 \mathrm{M} \mathrm{NaCl}$ and delivered by a variable pressure volumetric pump (model 560; IVAC Corp., San Diego, CA). The infusion was continued for $15 \mathrm{~h}$, until 2 a.m. the following morning (Fig. 1). Subjects were confined to bed

PROTOCOL FOR EXPERIMENT IN THE FASTED STATE

No meals given during the experiment

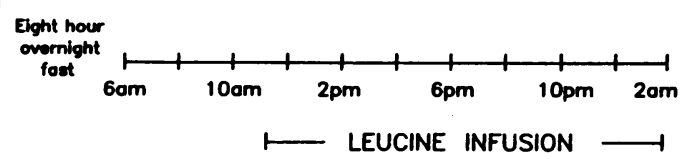

11111111111

PROTOCOL FOR EXPERIMENT IN THE FED STATE

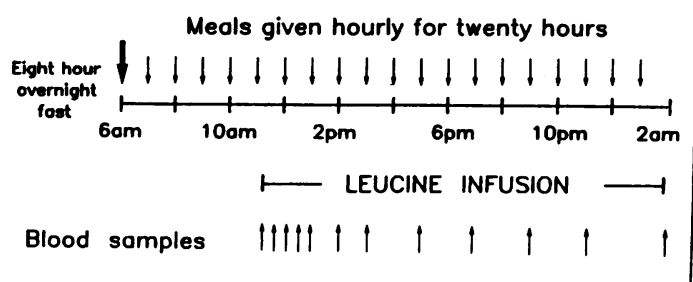

Figure 1. Protocol for the primed constant infusion experiments carried out in the fasted and fed states. For the experiment in the fasted state no food was given for the 20 -h duration of the study. For the experiment in the fed state a large meal was given at 6 a.m. (heavy arrow), representing $4 / 23$ of the daily calorie intake. Smaller meals which contained $1 / 23$ of daily calories were given every hour thereafter (smaller arrows). during the infusion, but were encouraged to sit up and maintain good circulation by periodically exercising their limbs. 15 -ml blood samples were drawn at designated times from an intravenous line attached to the opposite arm. Blood samples were taken before meals were eaten.

The protocol for the infusion carried out in the fasted state was identical to the one in the fed state; however, no meals were given. Subjects fasted for a total of $28 \mathrm{~h}$ ( 8 overnight and 20 during the course of the infusion). They were given free access to drinking water.

Isolation of lipoproteins. Blood was collected in tubes containing EDTA to give a final concentration of $0.1 \%$ EDTA. Plasma was separated by centrifugation $(2,500 \mathrm{rpm})$ for $20 \mathrm{~min}$ at $4^{\circ} \mathrm{C}$. Lipoproteins were isolated from plasma by sequential ultracentrifugation. VLDL were isolated from $5 \mathrm{ml}$ of plasma by a single ultracentrifugal spin $\left(39,000 \mathrm{rpm}, 18 \mathrm{~h}, 4^{\circ} \mathrm{C}\right)$ at a density of $1.006 \mathrm{~g} / \mathrm{ml}$ in a $50.3 \mathrm{Ti}$ rotor (Beckman Instruments, Inc., Palo Alto, CA). (In the fed state this fraction also contained chylomicrons. We have therefore referred to it previously $(7,8)$ as the triglyceride-rich lipoprotein fraction. Since we were interested in the apo B-100-containing lipoproteins of this fraction, we have referred to it in the present study as the VLDL fraction in - both the fasted and fed state.) Plasma infranates were adjusted to a density of $1.019 \mathrm{~g} / \mathrm{ml}$ with $\mathrm{KBr}$ and spun $\left(39,000 \mathrm{rpm}, 18 \mathrm{~h}, 4^{\circ} \mathrm{C}\right)$ to obtain the intermediate density lipoprotein (IDL) ${ }^{1}$ fraction. Infranates were in turn adjusted to $1.063 \mathrm{~g} / \mathrm{ml}$ and spun $\left(45,000 \mathrm{rpm}, 18 \mathrm{~h}, 4^{\circ} \mathrm{C}\right)$ to obtain $\mathrm{LDL}$ and then adjusted to $1.21 \mathrm{~g} / \mathrm{ml}$ for isolation $(49,000$ rpm, $24 \mathrm{~h}, 4^{\circ} \mathrm{C}$ ) of $\mathrm{HDL}$. Isolated lipoproteins were not recentrifuged. All lipoprotein fractions were adjusted to $3 \mathrm{ml}$ with normal saline. Lipoprotein-deficient plasmas $(d>1.21 \mathrm{~g} / \mathrm{ml})$ were adjusted to $4 \mathrm{ml}$. Undialyzed samples were aliquotted and stored frozen at $-70^{\circ} \mathrm{C}$ until time of analysis.

Plasma and VLDL fractions were assayed for total (free and esterified) cholesterol and triglyceride with an ABA-200 bichromatic analyzer (Abbott Diagnostics, South Pasadena, CA) using enzymatic reagents (9). HDL cholesterol was measured by analyzing the supernate obtained by precipitating a plasma aliquot with dextran sulfate- $\mathrm{Mg}^{2+}$, as described by Warnick et al. (10). Lipid assays were standardized through the Centers for Disease Control's Lipid Standardization Program.

Quantitation of apolipoproteins. Apo B was assayed with a noncompetitive ELISA (11). Apo A-I was assayed with the same system using apo A-I polyclonal antibodies. Plasma samples were diluted $1: 3,000$ for the apo B assay and 1:60,000 for the apo A-I assay. VLDL samples from fed and fasted individuals were diluted $1: 600$ and 1:300, respectively, for quantitation of apo $B$. VLDL samples were not pretreated in order to expose antigenic sites on apo B. Our assay was found to be unaffected by lipid in VLDL, possibly due to the detergent (Tween 20,0.05\%) used in all assay buffers. Furthermore, in our experience, incubation of VLDL samples with commercial lipases (shown in other systems to increase the amount of measurable apo B [12]) causes a decrease in the amount of apo B measured by ELISA, due to proteolytic degradation of VLDL apo B by contaminating proteases (13). At least three control plasmas were used on each plate as internal standards. ELISA assays were calibrated with reference material obtained from the Centers for Disease Control, Atlanta, GA.

The concentrations of plasma apo B, VLDL apo B, and IDL apo B were measured directly by adding diluted samples (in duplicate) to the microtiter plates. LDL apo $B$ was calculated by difference: plasma apo B - (VLDL apo B + IDL apo B). VLDL apo B concentration measurements (obtained by polyclonal ELISA assay) were taken to represent the concentration of VLDL apo B-100, since in the fasted or fed state $<3 \%$ of total VLDL apo B was apo B-48 (8). Plasma apo A-I concentrations were taken to represent the concentration of apo A-I in HDL, since $>95 \%$ of plasma apo A-I was HDL apo A-I. (In the fasted and fed state, the plasma concentration of apo A-I in the $d<1.006 \mathrm{~g} / \mathrm{ml}$ fraction is $<1 \mathrm{mg} / 100 \mathrm{ml}[8]$.)

1. Abbreviations used in this paper: ape, atom percent excess; FPR, fractional production rate; IDL, intermediate density lipoprotein. 
Isolation of apolipoproteins. Apo B-100 was isolated from VLDL and LDL and apo A-I from HDL by preparative SDS polyacrylamide gradient (4-22.5\%) gel electrophoresis as described previously (8). Lipoprotein samples were loaded by volume. Routinely, $200 \mu \mathrm{l}$ (50-100 $\mu \mathrm{g}$ protein) of VLDL or $100 \mu \mathrm{l}$ (100-150 $\mu \mathrm{g}$ protein) of LDL or HDL were loaded onto gels, which were run overnight $(16 \mathrm{~h})$ at $50 \mathrm{~V}$. Apolipoproteins were identified by comparing the distance they migrated into the gels with known molecular standards (high molecular weight standard mixture; Sigma Chemical Co., St. Louis, MO).

Measurement of stable isotope enrichment. Apolipoprotein bands were excised from polyacrylamide gels and hydrolyzed $\left(110^{\circ} \mathrm{C}, 24 \mathrm{~h}\right)$ in $6 \mathrm{~N} \mathrm{HCl}$ under nitrogen using a Pico-Tag work station (Waters Associates, Milford, MA). The hydrolyzates were chilled for $20 \mathrm{~min}$ at $-20^{\circ} \mathrm{C}$ and after centrifugation $(3,000 \mathrm{rpm}, 5 \mathrm{~min})$ the clear supernatant containing free amino acids was separated from precipitated polyacrylamide gel. Free leucine was extracted from the hydrolyzates using small $(5 \mathrm{~cm})$ Dowex 50x-8, 200-400 mesh cation exchange columns. Leucine was eluted from the columns with $3 \mathrm{~N} \mathrm{NH}_{4} \mathrm{OH}$. The sample was completely dried using a Speed-Vac evaporator (Savant Instruments, Inc., Hicksville, NY), and $1 \mathrm{ml} 3.5 \mathrm{M} \mathrm{HBr}$ in propanol was added. The reactants were heated $\left(30 \mathrm{~min}\right.$ at $\left.100^{\circ} \mathrm{C}\right)$ and the acid was then removed in the Speed-Vac evaporator. Heptafluorobutyric anhydride $(50 \mu \mathrm{l})$ and ethyl acetate were added and the samples were heated $\left(150^{\circ} \mathrm{C}\right.$ for $\left.15 \mathrm{~min}\right)$. Excess reagent was evaporated and $50 \mu \mathrm{l}$ ethyl acetate was added. Samples were analyzed with a 5985B gas chromatograph-mass spectrometer (Hewlett-Packard Co., Palo Alto, CA) using negative chemical ionization and methane as the reagent gas. Selective ion monitoring at $\mathrm{m} / \mathrm{z}=349$ and 352 (unlabeled and labeled leucine, respectively, were used to determine isotopic abundance. Enrichment of protein samples with $\left[\mathrm{D}_{3}\right]$ leucine was measured in atom percent excess (ape), calculated according to the formula:

enrichment (ape) $=\frac{I R_{t}-I_{0}}{\left(I R_{t}-I R_{0}\right)+1} \times 100$,

where $\mathrm{IR}_{t}=$ isotope ratio of sample at time point $t$, and $\mathrm{IR}_{0}=$ isotope ratio of sample at zero time (before $\left[D_{3}\right]$ leucine administration). Plasma leucine enrichment was measured by extracting the leucine on cation exchange columns and then following the same procedure as described for the apolipoprotein samples.

Calculation of apolipoprotein production. Apolipoprotein production was measured as the rate of incorporation of deuterated leucine into circulating apolipoproteins. This parameter, by definition, reflects the rate of apolipoprotein synthesis in the liver and also the rate of secretion of this protein into the circulation. The fractional production rate (FPR) for each apolipoprotein (measured in pools/day) was calculated as:

$\frac{\text { rate of increase of leucine enrichment in apolipoprotein (ape/h) }}{\text { enrichment of VLDL apo B-100 at plateau (ape) }} \times 24$,

where enrichment of VLDL apo B-100 at plateau was taken as the average of VLDL apo B-100 enrichment at the last three or four timepoints (depending on the shape of the curve). A linear rate of increase in apolipoprotein enrichment was calculated manually by using 4-5 timepoints (a period of $\sim 4 \mathrm{~h}$ ) for the VLDL curves (not always including the zero timepoint) and 5-6 timepoints (a period of $\sim 9 \mathrm{~h}$ ) for the LDL and HDL curves. Manual plots were not significantly different from lines of best fit obtained by least squares linear regression analysis. Absolute production rates (milligrams/kilogram per day) were calculated as:

$\frac{\text { FPR (pools/d) } \times \text { apolipoprotein pool size }(\mathrm{mg})}{\text { body weight }(\mathrm{kg})}$,

where apolipoprotein pool size $=$ plasma apolipoprotein concentration $(\mathrm{mg} / 100 \mathrm{ml}) \times$ plasma volume $(0.045 \mathrm{liter} / \mathrm{kg})$.

Statistical analysis. Data were tabulated and stored in a VAX-780 computer (Digital Equipment Co., Maynard, MA) using the scientific software package RS/1 (BBN Research Systems, Cambridge, MA).
Paired $t$ tests were used to assess the significance of individual mean differences. Repeated measures analysis of variance was used to assess the significance of repeated mean differences during the constant infusion experiments. Least squares linear regression analysis was used to test the manually obtained lines of best fit.

\section{Results}

Plasma lipid levels were monitored during the course of the stable isotope infusion experiments. In the fasted state, plasma triglyceride concentration remained constant, whereas in the fed state more variability was observed (Fig. 2). The protocol for the experiment in the fed state was designed to maintain the plasma concentration of triglyceride at as constant a level as physiologically possible; a steady-state condition was, however, difficult to achieve in all subjects. Plasma triglyceride concentration was nevertheless significantly greater in the fed compared with the fasted state $(154 \pm 18$ vs. $64 \pm 7 \mathrm{mg} / 100 \mathrm{ml}$, $P<0.001)$. Plasma cholesterol concentration did not change significantly during the infusion experiments and there was no significant difference between the cholesterol concentration in the fasted and fed states $(155 \pm 7$ vs. $147 \pm 7 \mathrm{mg} / 100 \mathrm{ml}$, respectively).

Mean VLDL triglyceride, cholesterol, and apo B, and HDL cholesterol concentrations are shown in Fig. 3. In the fed state, the VLDL fraction contained chylomicrons as well as VLDL, but for the sake of comparison it is always referred to in the present study as the VLDL fraction. The plasma concentration of all three VLDL components was significantly elevated in the fed vs. fasted state (VLDL triglyceride: $108 \pm 17$ vs. $35 \pm 6$ $\mathrm{mg} / 100 \mathrm{ml}, P<0.001$; VLDL cholesterol: $13.8 \pm 2.1$ vs. $5.9 \pm 1.3 \mathrm{mg} / 100 \mathrm{ml}, P<0.01$; VLDL apo B: $5.0 \pm 1.1$ vs. $3.1 \pm 1$ $\mathrm{mg} / 100 \mathrm{ml}, P<0.05)$. HDL cholesterol concentration was significantly decreased ( $38 \pm 4$ vs. $42 \pm 4 \mathrm{mg} / 100 \mathrm{ml}, P<0.05)$. These differences in the plasma concentration of VLDL and HDL components are very similar to the postprandial changes, which we observed in healthy human subjects fed a single fat-rich meal (8).

Plasma leucine enrichment (measured in atom percent excess) increased rapidly after the initial bolus injection of isotope and remained relatively constant for the 15-h duration of the studies (Fig. 4). Mean plasma leucine enrichment was significantly less in the fed compared with the fasted state
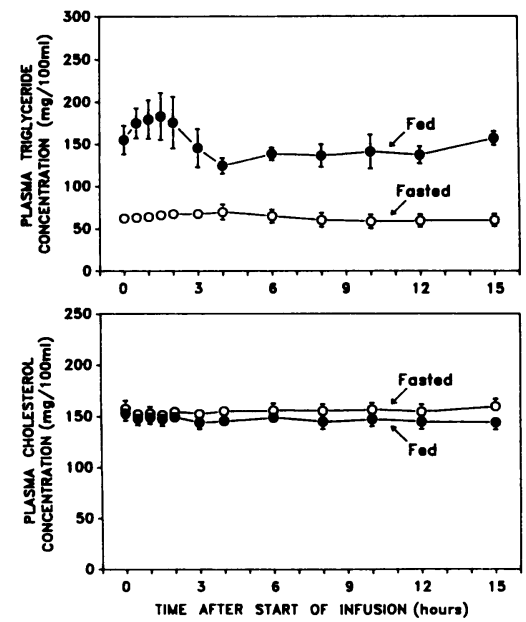

Figure 2. Plasma triglyceride and cholesterol concentration of subjects during the constant infusion experiments in the fasted (open circles) and fed state (solid circles). Values represent means \pm SEM for six subjects. 

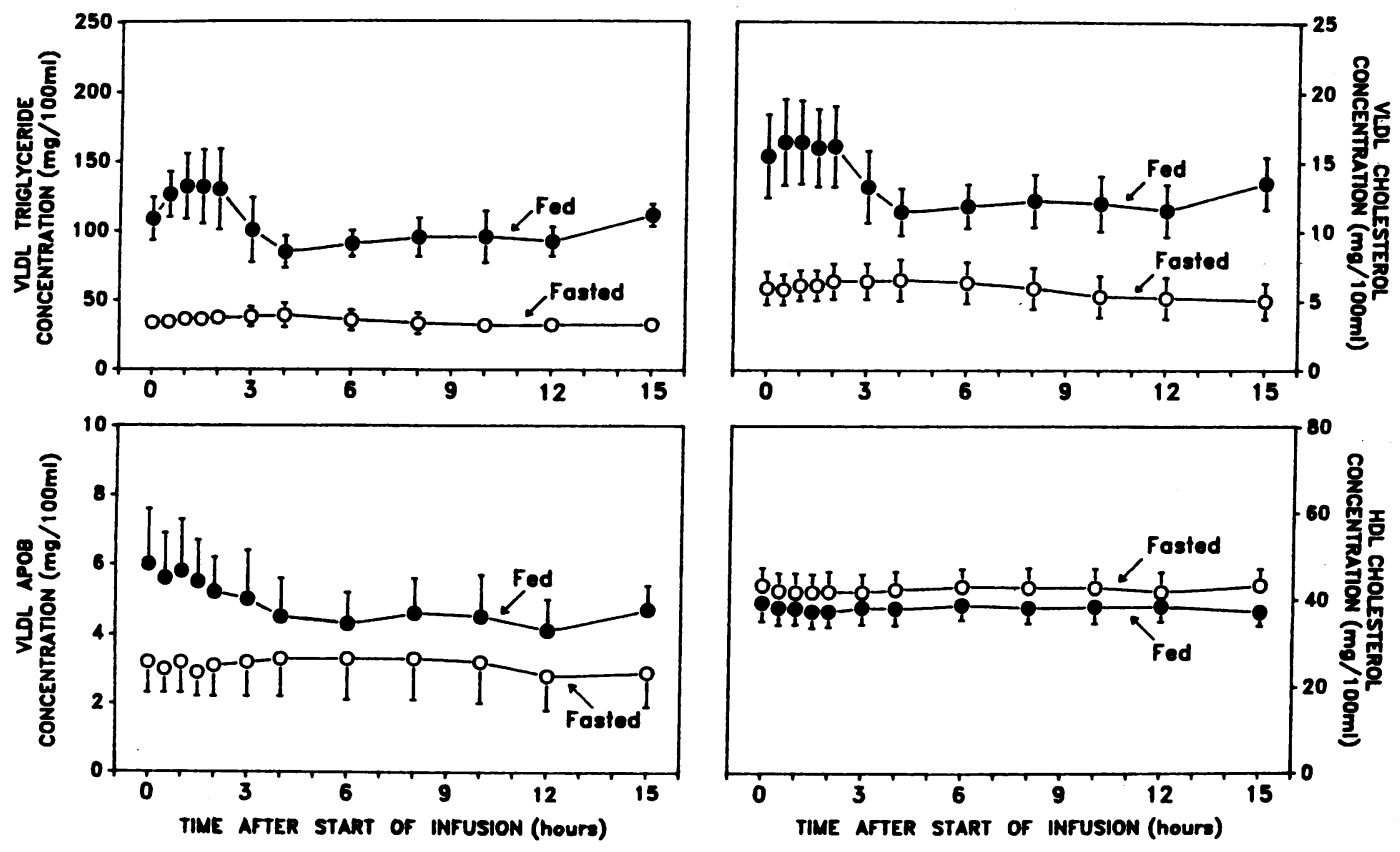

Figure 3. Plasma concentration of VLDL triglyceride, cholesterol, and apo B, and HDL cholesterol in subjects during the constant infusion experiments in the fasted (open circles) and fed state (solid circles). Values represent meáns \pm SEM for six subjects.

(6.64 \pm 0.51 vs. $7.97 \pm 0.27$ ape, $P<0.05)$. Since the same amount of stable isotope was given during the fasted and fed experiments, the plasma enrichment data suggested that plasma leucine concentrations must have been different during the two experiments. This was confirmed by amino acid analysis of plasma samples obtained at the time of stable isotope administration ( $5 \mathrm{~h}$ after the start of feeding; Fig. 1). The mean concentrations of all amino acids were greater in the fed state compared with the fasted state (leucine: $148 \pm 8$ vs. $114 \pm 5$ $\mu \mathrm{mol} /$ liter, $P<0.01$ ).

The separation of VLDL apolipoproteins on 4-22.5\% polyacrylamide gradient gels is shown in Fig. 5 for samples obtained from a single subject. The apolipoprotein composition of VLDL during the infusion experiments remained relatively constant, as determined by the consistent banding pattern of the VLDL samples. LDL and HDL lipoprotein samples were also separated on polyacrylamide gradient gels; apo B-100 was essentially the only protein in LDL and apo A-I was the major protein in HDL. In some LDL samples, characteristic apo B-100 fragments (e.g., apo B-76 and apo B-24 [14])

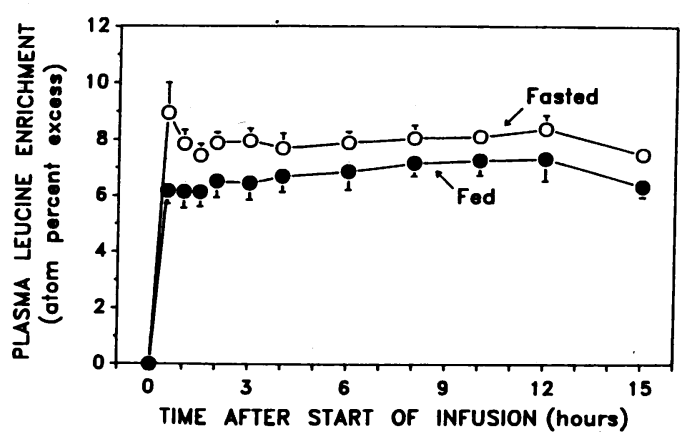

Figure 4. Enrichment of plasma leucine with deuterated leucine during the constant infusion experiments in the fasted (open circles) and fed state (solid circles). Values represent means \pm SEM for six subjects. were observed; however, only protein that migrated with typical apo B-100 mobility was used for measurement of isotopic enrichment.

As shown in Fig. 6, deuterated leucine enrichment of VLDL apo B-100 increased linearly soon after the priming dose of stable isotope. A plateau in VLDL apo B-100 enrichment was observed in both the fasted and fed states 6-8 $\mathrm{h}$ after the start of the infusion. The enrichment of VLDL apo B-100 was significantly less in the fed state. VLDL apo B-100 enrichment curves (Fig. 6) plateaued at levels less than the plasma leucine enrichment curves (Fig. 4), reflecting the difference in enrichment of leucine in the plasma and tissue (precursor amino acid) pools.

The enrichment of LDL apo B-100 and HDL apo A-I during the fasted and fed experiments is shown in Fig. 7. Measurable enrichment of these two proteins occurred 2-3 $\mathrm{h}$ after the start of the infusion in most subjects. In some cases, however, measurable enrichment was not observed until 8-10 h after the start of the infusion; enrichment was not seen at earlier time points due to the limited sensitivity of the gas chromatograph/mass spectrograph instrument. The enrichment of these proteins with deuterated leucine tended to be linear over time, and tended to be less in the fed compared with the fasted state (Fig. 7).

To calculate plasma apolipoprotein pool sizes, apolipoprotein concentrations for each subject were calculated to be the average of 12 measurements obtained during the course of the infusion experiments. VLDL apo B was significantly greater $(5 \pm 1: 1$ vs. $3.1 \pm 0.9 \mathrm{mg} / 100 \mathrm{ml}, P<0.05)$, whereas IDL apo B was significantly less $(0.6 \pm 0.1$ vs. $1.1 \pm 0.3 \mathrm{mg} / 100 \mathrm{ml}, P$ $<0.05$ ) in the fed compared with the fasted state. Plasma apo B, LDL apo B, and plasma apo A-I concentrations were not significantly different in the fasted and fed states $(66 \pm 8$ vs. $63 \pm 8 \mathrm{mg} / 100 \mathrm{ml}, 62 \pm 7$ vs. $58 \pm 7 \mathrm{mg} / 100 \mathrm{ml}$, and $124 \pm 9$ vs. $125 \pm 7 \mathrm{mg} / 100 \mathrm{ml}$, respectively).

Parameters of VLDL apo B-100, LDL apo B-100, and HDL apo A-I production are shown in Table I. Absolute 

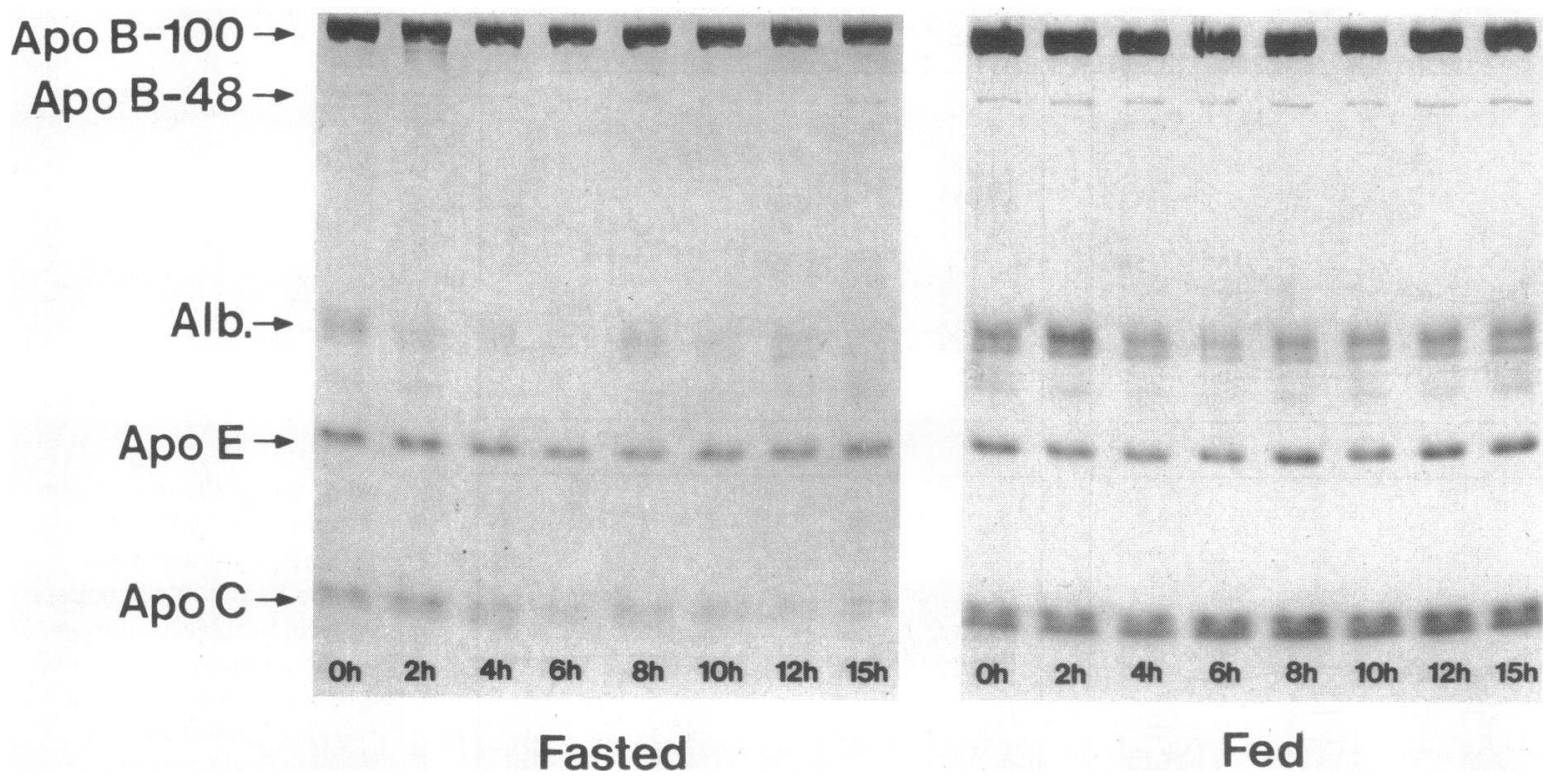

Fed

Figure 5. Polyacrylamide gradient gel electrophoretic separation of VLDL apolipoproteins, isolated at the time points indicated from a single subject during the fasted and fed experiments. Five major protein bands are indicated on the left of the figure. Alb., albumin.

VLDL apo B-100 production was significantly greater ( $P$ $<0.01)$ and LDL apo B-100 production was significantly less $(P<0.05)$ in the fed vs. the fasted state. This reduction in LDL apo B-100 production was offset by a concommitant significant decrease $(P<0.01)$ in the fractional production rate of fractional catabolic rate of $\mathrm{LDL}$ apo $\mathrm{B}-100$ in the fed vs. the fasting state. LDL apo B-100 production was significantly greater $(P<0.01)$ than VLDL apo B-100 production in the fasted state, and tended to be less (not statistically significant) in the fed state. Production rates obtained using the present technique were comparable to those obtained by previous methods $(15,16)$ (Table I).

To see whether there was any relationship between absolute rates of apo B-100 and apo A-I production and the plasma concentration of particular lipoprotein lipids, correlation analyses were carried out using data from all 14 infusion experiments ( 7 in the fasted state and 7 in the fed state). VLDL apo B-100 production rates were significantly $(P<0.01)$ correlated $(r=0.73)$ with VLDL triglyceride levels. LDL apo B-100 production rates were significantly $(P<0.01)$ correlated $(r=0.76)$ with LDL cholesterol levels. The correlation between HDL

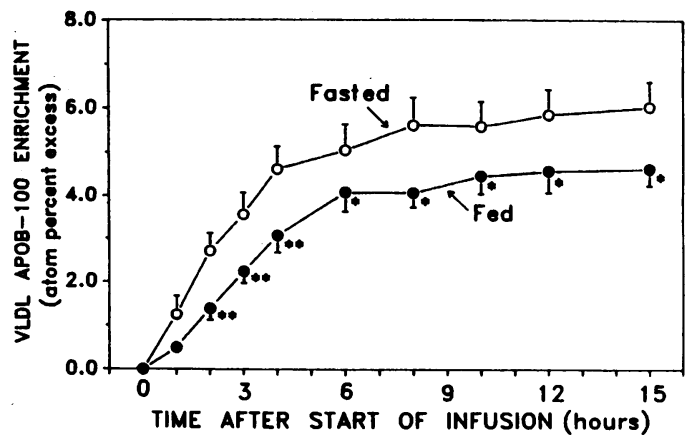

Figure 6. Enrichment of VLDL apo B-100 with deuterated leucine during the fasted (open circles) and fed experiments (solid circles). Values represent means \pm SEM for six subjects. apo A-I production rates and HDL cholesterol levels $(r=0.42)$ was not, however, statistically significant $(P=0.13)$.

\section{Discussion}

A number of methods for measuring protein synthesis in vivo have been described, involving the administration of isotopically labeled amino acids and the subsequent measurement of their incorporation into specific proteins (17). The main difficulty with these methods has been the assessment of specific activity or enrichment of the precursor amino acid pool at the site of protein assembly, an important requirement for the accurate measurement of protein synthesis. The direct measurement of aminoacyl-tRNA is difficult and impractical, and amino acids from the more readily accessible compartments, such as the plasma or total intracellular pools, cannot be used reliably because their specific radioactivities or enrichments may differ from each other and from that of aminoacyl-tRNA $(18,19)$.

The rapid turnover of VLDL apo B-100 and the measurable amounts of this protein in plasma provide a unique op-

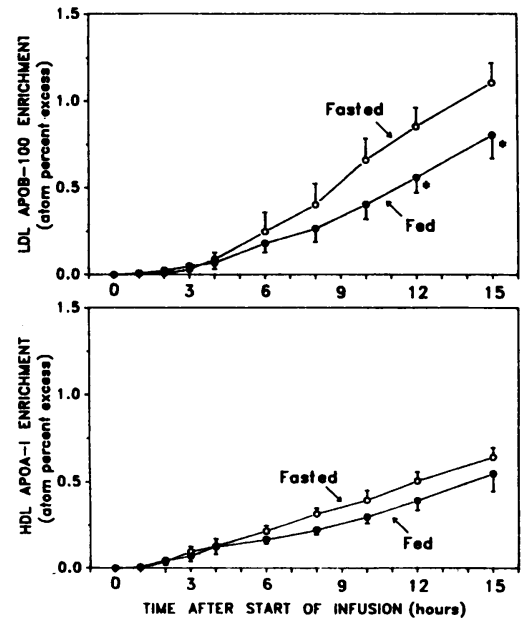

Figure 7. Enrichment of LDL apo B-100 and HDL apo A-I with deuterated leucine during the fasted (open circles) and fed experiments (solid circles). Values represent means \pm SEM for six subjects. 
Table I. Parameters of VLDL apo B-100, LDL apo B-100, and HDL apo A-I Production in the Fasted and Fed State

\begin{tabular}{lcccc}
\hline & $\begin{array}{c}\text { Rate of } \\
\text { enrichment }\end{array}$ & FPR & Pool size & $\begin{array}{c}\text { Absolute } \\
\text { production } \\
\text { rate }\end{array}$ \\
\hline & & & & $m g / k g$ \\
& ape/d & fractions $/ d$ & $m g$ & per $d$ \\
Fasted & & & & \\
$\quad$ VLDL apo B-100 & $29.4 \pm 4.1$ & $5.13 \pm 0.67$ & $103 \pm 31$ & $6.4 \pm 1.7$ \\
LDL apo B-100 & $2.72 \pm 0.24$ & $0.48 \pm 0.03$ & $2,042 \pm 241$ & $13.1 \pm 1.2$ \\
HDL apo A-I & $1.18 \pm 0.06$ & $0.22 \pm 0.02$ & $4,010 \pm 264$ & $11.7 \pm 1.1$ \\
Fed & & & & \\
VLDL apo B-100 & $20.6 \pm 2.0^{*}$ & $4.64 \pm 0.40$ & $163 \pm 37^{*}$ & $9.9 \pm 1.7^{\ddagger}$ \\
LDL apo B-100 & $1.56 \pm 0.17^{\ddagger}$ & $0.34 \pm 0.01^{\ddagger}$ & $1,901 \pm 252$ & $8.9 \pm 1.2^{*}$ \\
HDL apo A-I & $0.82 \pm 0.12^{*}$ & $0.18 \pm 0.02$ & $4,031 \pm 223$ & $10.1 \pm 1.2$ \\
& & & & \\
\hline
\end{tabular}

Values represent means \pm SEM for six subjects.

Kinetic parameters were calculated as described in Methods.

VLDL apo B-100 enrichment at plateau was $5.78 \pm 0.57$ ape in the fasted state and $4.55 \pm 0.44$ ape in the fed state $(P<0.05)$.

Significantly different from the fasted state by paired $t$ test: ${ }^{*} P<0.05$, $¥ P<0.01$.

Range of production rates obtained by previous studies (reviewed in references 15 and 16 ). VLDL apo B: $9.1-15.3 \mathrm{mg} / \mathrm{kg}$ per d; LDL apo B: $7.6-14.4 \mathrm{mg} / \mathrm{kg}$ per d; HDL apo A-I: $8.7-15.8 \mathrm{mg} / \mathrm{kg}$ per $\mathrm{d}$.

portunity to estimate the enrichment of the hepatic precursor amino acid pool. In an isotopic steady state the enrichment of a product should eventually reach the enrichment of its precursor. The validity of this approach to measure apolipoprotein production (using a constant infusion of deuterated leucine) is, however, dependent on certain assumptions. First, it must be assumed that all cells in the liver with the capacity to synthesize apolipoproteins are labeled equally, and that intracellular leucine pools are labeled rapidly and homogeneously. The rapid equilibration of plasma leucine and tissue leucyltRNA pools in the rat $(20,21)$ supports the use of labeled leucine as an appropriate tracer in this regard. Second, it must be assumed that pools of apo B-100 in the liver are homogeneously labeled and that essentially all newly synthesized apo B-100 is secreted. In vitro experiments in the rat suggest that a proportion of apo B synthesized in the liver is degraded intracellularly and is not secreted (22); however, it remains to be determined if this is a quantitatively important pathway in man. For the sake of the present method, it must be assumed that intracellular degradation is minimal. A further assumption is that hepatic apolipoproteins are derived from a common pool of amino acids and that the circulating apolipoproteins that are isolated from plasma are indeed of hepatic origin. Evidence has been presented to show that the intestine has the capacity to synthesize apo B-100 (23); however, the majority of circulating apo B-100 is probably of hepatic origin. Furthermore, it is well known that the intestine can synthesize and secrete apo A-I (24). For our whole-body apo A-I production rates to be valid it must be assumed that the enrichment of hepatic and intestinal tissues under the present experimental conditions were similar, or that the majority of plasma apo A-I in man is derived from the liver. This latter assumption is probably true in the fasted state, though in the fed state, intestinal production of apo A-I is more significant. Our HDL apo A-I production rates in the fed state must therefore be interpreted with caution.

A stable isotope method for measuring VLDL apo B synthesis (more correctly termed production) has recently been published by Cryer et al. (5). Our approach is, however, different from this method in several ways. First, we used the stable isotope $\left[\mathrm{D}_{3}\right] \mathrm{L}$-leucine, instead of $\left[{ }^{15} \mathrm{~N}\right]$ glycine. Measurable incorporation of amino acid into apolipoproteins is thus optimized, since apo B-100 and apo A-I contain more leucine than any other amino acid $(14,25)$. Second, we used the plateau enrichment of VLDL apo B-100 to estimate the enrichment of the tissue amino acid precursor pool, instead of the plateau enrichment of urinary hippurate. This is an advantage, since it avoids the need to collect and analyze urinary hippurate samples. It also provides a more accurate measure of the enrichment of the amino acid precursor pool, since apo B-100 is a more direct product derived from intracellular amino acid pools than hippurate. Third, polyacrylamide gradient gel electrophoresis was used to isolate apolipoproteins, which was a fast and efficient way to prepare pure protein samples at multiple timepoints. Fourth, stable isotope was infused for a 15-h rather than an 8-h period (5). Plateau enrichment in VLDL apo B-100 was therefore better defined, and enrichment of deuterated leucine in the slowly turning over LDL apo B-100 and HDL apo A-I pools was more easily detected.

The most unique feature of this study is that apolipoprotein production, for the first time, has been measured in human subjects in the fasted and fed state. Lipoprotein kinetic experiments in the past have always been carried out over at least a 1-2-d period. Subjects have therefore been studied during periods of both fasting and feeding. In previous VLDL apo B kinetic studies (26-30), fat- and calorie-restricted diets have been given to minimize the secretion of chylomicrons and VLDL from the intestine. This rather artificial situation, however, represents neither a fasted nor fed state. Since we have studied subjects over a shorter period of time, during wellcontrolled periods of either fasting or feeding, we believe that the present method represents a more physiological approach to the study of apolipoprotein kinetics.

We have found that feeding caused an increase in VLDL apo B-100 production and a decrease in LDL apo B-100 production (Table I). In the fed state, VLDL apo B-100 production tended to be greater than LDL apo B-100 production, whereas in the fasted state LDL apo B-100 production was significantly greater than VLDL apo B-100 production. These results need to be interpreted in relation to our current understanding of apo B-100 metabolism. Triglyceride-rich, apo B-100-containing lipoproteins are secreted from the liver (arrow 1, Fig. 8) and are depleted of triglyceride in the circulation by lipoprotein lipase. VLDL are converted to IDL, which are in turn delipidated (arrow 3, Fig. 8) to form LDL. The apo B-containing lipoproteins in plasma are thus part of a catabolic cascade, forming a spectrum of different-sized lipoprotein particles. Original studies showed that a precursor-product relationship existed between VLDL and LDL in normolipidemic individuals (26-28) (i.e., all VLDL was converted to LDL, and all LDL was derived from VLDL). In more recent studies (30-33), however, it has been shown that a significant proportion of VLDL apo B can be cleared from the plasma by receptor-mediated uptake (presumably in the liver) (arrow 2, Fig. 8) and is therefore not converted to LDL. Furthermore, it has been suggested that as much as one-third of LDL apo B is not derived from VLDL in normolipidemic individuals (described as direct input of LDL (31) (arrow 4, 'Fig. 8). In this study we measured LDL apo B-100 production as the rate of incorporation of deuterated leucine into apo B-100 in the $1.019-1.063 \mathrm{~g} / \mathrm{ml}$ density fraction of plasma. This represents 


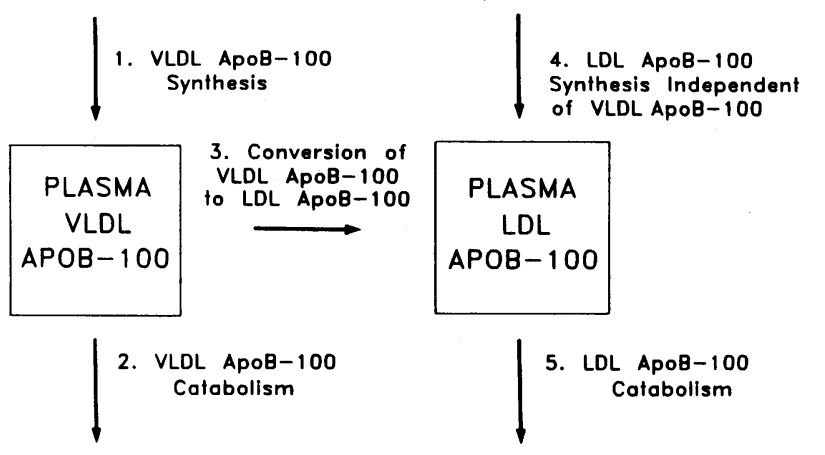

Figure 8. Diagrammatic representation of plasma apo B-100 metabolism showing the VLDL and LDL pools of plasma apo B-100. Arrows 1 and 4 represent the entry of newly synthesized apo B-100 into the system, and arrows 2 and 5 represent the tissue uptake and catabolism of apo B-100. Arrow 3 represents the conversion of VLDL apo B-100 to LDL apo B-100, due to lipoprotein lipolysis in the circulation. In the fed state, VLDL apo B-100 production (1) and catabolism (2) is increased, and conversion (3) is probably diminished. In the fasted state LDL apo B-100 production independent of VLDL apo B-100 (4) is increased, conversion (3) is probably also enhanced, and apo B-100 catabolism (5) is increased.

newly synthesized apo B-100, which was converted from VLDL apo B-100 to LDL apo B-100 (arrow 3), and also newly synthesized apo B-100, produced independently of VLDL (arrow 4). A proportion of this apo B-100 is possibly secreted on IDL-sized lipoproteins, which are catabolized to LDL due to rapid, first pass conversion (31). Since LDL apo B-100 production was significantly greater than VLDL apo B-100 production in the fasted state, our data suggest that a considerable proportion (at least 50\%) of LDL apo B-100 was synthesized independently of VLDL in the fasted state. Even if one assumes that all VLDL apo B-100 was catabolized to LDL apo B-100 in the fasted state, then $6.4 \mathrm{mg} / \mathrm{kg}$ per $\mathrm{d}$ of LDL apo B-100 production was due to the conversion of VLDL apo B- 100 , and $6.7 \mathrm{mg} / \mathrm{kg}$ per d $(13.1-6.4 \mathrm{mg} / \mathrm{kg}$ per d) was due to direct LDL apo B-100 production. Direct input of LDL apo B-100 was not evidenced in the fed state, since VLDL apo B-100 production tended to be greater than LDL apo B-100 production. This circumstantial evidence does not, however, exclude the possibility that in the fed state a small proportion of LDL apo B-100 may also have been synthesized independently of VLDL.

Although the present data and that of Kesaniemi et al. (32) have provided direct evidence for the independent production of LDL in normolipidemic humans, numerous studies in experimental animals or in isolated cells in culture have previously documented the liver's ability to secrete LDL-sized lipoproteins (34-39). Studies have shown that the extent of hepatic LDL and VLDL secretion is dependent on the lipid status of the liver. Perfused livers from fed pigs (40) and rats (41) have been shown to secrete more VLDL apo B and less LDL apo B than livers from fasted animals. Similar effects have been observed in isolated hepatocytes from fed and fasted rats (42). In human Hep G2 cells supplemented with free fatty acids, inclusion of oleic acid in cell media caused $\left[{ }^{35} \mathrm{~S}\right] \mathrm{methio}-$ nine incorporation into VLDL to increase and into LDL to decrease (43). Redistribution of apo B secretion from LDL to VLDL, due to the addition of various fatty acids complexed to BSA, has also been observed in the colonic adenocarcinoma cell line Caco-2 (44). It is significant that the present study for the first time provides in vivo evidence to support these in vitro observations. Our data suggest that in humans, in the fed state, apo B-100 is secreted on larger triglyceride-rich lipoproteins, and is thus found in the VLDL $(d<1.006 \mathrm{~g} / \mathrm{ml})$ fraction of plasma. In the fasted state, however, smaller particles are produced, which contain less triglyceride, and newly synthesized apo B-100 is consequently found in LDL in the 1.019$1.063 \mathrm{~g} / \mathrm{ml}$ fraction of plasma. Direct production of VLDL and LDL is thus reciprocally related, and is dependent on the lipid status of the liver, a concept supported by studies in hypertriglyceridemic subjects before and after weight loss, in which direct secretion of LDL apo B was inversely related to the rate of VLDL triglyceride secretion (45).

In conclusion, our data suggest that in the fed state VLDL apo B-100 production is increased, direct clearance of VLDL apo B-100 by the liver is enhanced, and conversion to LDL apo B-100 (through IDL) is reduced. There is, therefore, a greater flux of apo B-100 through the arrowed pathways 1 and 2 in Fig. 8. The plasma concentration of VLDL apo B-100 is subsequently increased. In the fasted state there is a greater flux of apo B-100 through pathways 3, 4, and 5. Total LDL apo B-100 production is increased due to increased direct LDL apo B-100 production and possibly to enhanced conversion of VLDL apo B-100. LDL apo B-100 catabolism is in turn increased (probably due to a change in receptor activity), resulting in no significant increase in LDL apo B-100 concentration.

Further studies are being carried out to extend this method to the measurement of other plasma apolipoproteins and to determine the viability of using other stable isotope amino acids.

\section{Acknowledgments}

This work was sponsored by grant HL-39326 from the National Institutes of Health and contract 53-3K06-1-10 from the United States Department of Agriculture Research Service.

\section{References}

1. Castelli, W. P., R. J. Garrison, P. W. F. Wilson, R. D. Abbott, S. Kalousdian, and W. B. Kannel. 1986. Incidence of coronary heart disease and lipoprotein cholesterol levels: the Framingham study. JAMA (J. Am. Med. Assoc.). 256:2835-2838.

2. 1982. Lipoprotein Kinetics and Modeling. M. Berman, S. M. Grundy, and B. V. Howard, editors. Academic Press, Inc. New York.

3. Eaton, R. P., R. C. Allen, and D. S. Schade. 1982. Beta-apolipoprotein secretion in man: investigation by analysis of ${ }^{75} \mathrm{Se}$-amino acid incorporation into apoprotein. In Lipoprotein Kinetics and Modeling. M. Berman, S. M. Grundy, and B. V. Howard, editors. Academic Press, Inc. New York. 77-97.

4. Fisher, W. R., L. A. Zech, P. Bardalaye, G. Warmke, and M. Berman. 1980. The metabolism of apolipoprotein B in subjects with hypertriglyceridemia and polydisperse LDL. J. Lipid Res. 21:760-774.

5. Cryer, D. R., T. Matsushima, J. B. Marsh, M. Yudkoff, P. M. Coates, and J. A. Cortner. 1986. Direct measurement of apolipoprotein B synthesis in human very low density lipoprotein using stable isotopes and mass spectrometry. J. Lipid Res. 27:508-516.

6. Ordovas, J. M., L. Litwack-Klein, P. W. F. Wilson, M. M. Schaefer, and E. J. Schaefer. 1987. Apolipoprotein E isoform phenotyping methodology and population frequency with identification of apo E1 and E5 isoforms. J. Lipid Res. 28:371-380.

7. Cohn, J. S., J. R. McNamara, S. D. Cohn, J. M. Ordovas, and E. J. Schaefer. 1988. Postprandial plasma lipoprotein changes in human subjects of different ages. J. Lipid Res. 29:469-479.

8. Cohn, J. S., J. R. McNamara, S. D. Cohn, J. M. Ordovas, and E. J. Schaefer. 1988. Plasma apolipoprotein changes in the triglycer- 
ide-rich lipoprotein fraction of human subjects fed a fat-rich meal. $J$. Lipid Res. 29:925-936.

9. McNamara, J. R., and E. J. Schaefer. 1987. Automated enzymatic standardized lipid analyses for plasma and lipoprotein fractions. Clin. Chim. Acta. 166:1-8.

10. Warnick, G. R., J. Benderson, and J. J. Albers. 1982. Dextran sulfate- $\mathrm{Mg}^{++}$precipitation procedure for quantitation of high density lipoprotein cholesterol. Clin. Chem. 28:1379-1388.

11. Ordovas, J. M., J. Peterson, P. Santaniello, J. S. Cohn, P. W. F. Wilson, and E. J. Schaefer. 1987. Enzyme linked immunosorbent assay for human plasma apolipoprotein B. J. Lipid Res. 28:12161224.

12. Reardon, M. F., M. E. Poapst, K. D. Uffelman, and G. Steiner. 1981. Improved method for quantitation of B apoprotein in plasma lipoproteins by electroimmunoassay. Clin. Chem. 27:829-835.

13. Cohn, J. S., and E. J. Schaefer. 1987. Sample lipolysis for quantitation of apolipoprotein B. Clin. Chem. 33:338-339.

14. Kane, J. P., D. A. Hardman, and H. E. Paulus. 1980. Heterogeneity of apolipoprotein B: isolation of a new species from human chylomicrons. Proc. Natl. Acad. Sci. USA. 77:2465-2469.

15. Kesaniemi, Y. A., G. L. Vega, and S. M. Grundy. 1982. Kinetics of apolipoprotein $\mathrm{B}$ in normal and hyperlipidemic man: review of current data. In Lipoprotein Kinetics and Modeling. M. Berman, S. M. Grundy, and B. V. Howard, editors. Academic Press, Inc., New York. 181-205.

16. Schaefer, E. J., and J. M. Ordovas. 1986. Metabolism of apolipoproteins A-I, A-II, and A-IV. Methods Enzymol. 129:420-443.

17. Waterlow, J. C., P. J. Garlick, and D. J. Millward. 1978. Protein Turnover in Mammalian Tissues and in the Whole Body. North-Holland Publishing Co., Amsterdam.

18. Fern, E. B., and P. J. Garlick. 1973. The specific radioactivity of the precursor pool for estimates of the rate of protein synthesis. Biochem. J. 134:1127-1130.

19. Vidrich, A., J. Airhart, M. K. Bruno, and E. A. Khairallah. 1977. Compartmentation of free amino acids for protein bioproduction: influence of diurnal changes in hepatic amino acid concentrations on the composition of the precursor pool charging aminoacyltransfer ribonucleic acid. Biochem. J. 162:257-266.

20. Everett, A. W., G. Prior, and R. Zak. 1981. Equilibration of leucine between the plasma compartment and leucyl-tRNA in the heart, and turnover of cardiac myosin heavy chain. Biochem. $J$. 194:365-368.

21. Kelley, J., W. S. Stirewalt, and L. Chrin. 1984. Protein production in rat lung: measurement in vivo based on leucyl-tRNA and rapidly turning-over procollagen I. Biochem. J. 222:77-83.

22. Borchardt, R. A., and R. A. Davis. 1987. Intrahepatic assembly of very low density lipoproteins. Rate of transport out of the endoplasmic reticulum determines rate of secretion. J. Biol. Chem. 262:16394-16402.

23. Higuchi, K., A. V. Hospattankar, S. W. Law, N. Meglin, J. Cortright, and H. B. Brewer, Jr. 1988. Human apolipoprotein B (apoB) mRNA: identification of two distinct apoB mRNAs, an mRNA with the apoB-100 sequence and an apoB mRNA containing a premature in-frame translational stop codon, in both liver and intestine. Proc. Natl. Acad. Sci. USA. 85:1772-1776.

24. Glickman, R. M., and P. H. R. Green. 1977. The intestine as a source of apolipoprotein A-I. Proc. Natl. Acad. Sci. USA. 74:25692573.

25. Brewer, H. B., Jr., T. Fairwell, A. LaRue, R. Ronan, A. Houser, and T. J. Bronzert. 1978. The amino acid sequence of human apo A-I, an apolipoprotein isolated from high density lipoproteins. Biochem. Biophys. Res. Commun. 80:623-630.

26. Sigurdsson, G., A. Nicoll, and B. Lewis. 1975. Conversion of very low density lipoprotein to low density lipoprotein. A metabolic study of apolipoprotein B kinetics in human subjects. J. Clin. Invest. 56:1481-1490.

27. Berman, M., M. Hall III, R. I. Levy, S. Eisenberg, D. W Bilheimer, R. D. Phair, and R. H. Goebel. 1978. Metabolism of apo B and apo $\mathrm{C}$ lipoproteins in man: kinetic studies in normal and hyperlipoproteinemic subjects. J. Lipid Res. 19:38-56.

28. Reardon, M. F., N. H. Fidge, and P. J. Nestel. 1978. Catabolism of very low density lipoprotein B apoprotein in man. J. Clin. Invest. 61:850-860.

29. Melish, J., N. A. Le, H. Ginsberg, D. Steinberg, and W. V. Brown. 1980. Dissociation of apoprotein B and triglyceride production in very-low-density lipoproteins. Am. J. Physiol. 239:E354-E362.

30. Packard, C. J., A. Munro, A. R. Lorimer, A. M. Gotto, and J. Shepherd. 1984. Metabolism of apolipoprotein B in large triglyceriderich very low density lipoproteins of normal and hypertriglyceridemic subjects. J. Clin. Invest. 74:2178-2192.

31. Beltz, W. F., Y. A. Kesaniemi, B. V. Howard, and S. M. Grundy. 1985. Development of an integrated model for analysis of the kinetics of apolipoprotein B in plasma very low density lipoproteins, intermediate density lipoproteins, and low density lipoproteins. $J$. Clin. Invest. 76:575-585.

32. Kesaniemi, Y. A., W. F. Beltz, and S. M. Grundy. 1985. Comparison of metabolism of apolipoprotein B in normal subjects, obese patients, and patients with coronary heart disease. J. Clin. Invest. 76:586-595.

33. Egusa, G., W. F. Beltz, S. M. Grundy, and B. V. Howard. 1985 Influence of obesity on the metabolism of apolipoprotein $B$ in humans. J. Clin. Invest. 76:596-603.

34. Illingworth, D. R. 1975. Metabolism of lipoproteins in nonhuman primates: studies on the origin of low density lipoprotein apoprotein in the plasma of the squirrel monkey. Biochim. Biophys. Acta. 388:38-51.

35. Nakaya, N., B. H. Chung, J. R. Patsch, and O. D. Taunton. 1977. Synthesis and release of low density lipoproteins by the isolated perfused pig liver. J. Biol. Chem. 252:7530-7533.

36. Fidge, N. H., and P. Poulis. 1978. Metabolic heterogeneity in the formation of low density lipoprotein from very low density lipoprotein in the rat: evidence for the independent production of a low density lipoprotein subfraction. J. Lipid Res. 19:342-349.

37. Johnson, F. L., R. W. St. Clair, and L. L. Rudel. 1983. Studies on the production of low density lipoproteins by perfused livers from nonhuman primates. Effect of dietary cholesterol. J. Clin. Invest. 72:221-236

38. Huff, M. W., and D. E. Telford. 1985. Direct synthesis of low-density lipoprotein B in the miniature pig. Metab. Clin. Exp. 34:36-42.

39. Bell-Quint, J., and T. Forte. 1981. Time-related changes in the synthesis and secretion of very low density, low density and high density lipoproteins by cultured rat hepatocytes. Biochim. Biophys. Acta. 663:83-98.

40. Nakaya, N., B. H. Chung, and O. D. Taunton. 1977. Synthesis of plasma lipoproteins by the isolated perfused liver from the fasted and fed pig. J. Biol. Chem. 252:5258-5261.

41. Marsh, J. B., and C. E. Sparks. 1982. The effect of fasting on the secretion of lipoproteins and two forms of apo $B$ by perfused rat liver. Proc. Soc. Exp. Biol. Med. 170:178-181.

42. Davis, R. A., J. R. Boogaerts, R. A. Borchardt, M. MaloneMcNeal, and J. Archambault-Schexnayder. 1985. Intrahepatic assembly of very low density lipoproteins: varied synthetic response of individual apolipoproteins to fasting. J. Biol. Chem. 260:14137-14144.

43. Ellsworth, J. L., S. K. Erickson, and Allen D. Cooper. 1986. Very low and low density lipoprotein synthesis and secretion by the human hepatoma cell line Hep-G2: effect of free fatty acid. J. Lipid Res. 27:858-874.

44. Hughes, T. E., J. M. Ordovas, and E. J. Schaefer. 1988. Regulation of intestinal apolipoprotein B synthesis and secretion by Caco-2 cells: lack of fatty acid effects and control by intracellular calcium ion. J. Biol. Chem. 263:3425-3431.

45. Ginsberg, H. N., N.-A. Le, and J. C. Gibson. 1985. Regulation of the production and catabolism of plasma low density lipoproteins in hypertriglyceridemic subjects. J. Clin. Invest. 75:614-623. 